\title{
Expectativas e Beber Problemático entre Universitários ${ }^{1}$
}

\author{
Ana Carolina Peuker ${ }^{2}$ \\ Janaina Fogaça \\ Lisiane Bizarro \\ Universidade Federal do Rio Grande do Sul
}

\begin{abstract}
RESUMO - O beber problemático é recorrente entre universitários e está associado a inúmeras consequiências negativas. Portanto, é importante compreender os fatores de risco para este fenômeno. Examinou-se a relação entre expectativas sobre os efeitos do álcool e o padrão de beber de risco em universitários. Os participantes foram 165 universitários, com média de 22 anos $(d p=2,5)$ que responderam aos inventários AUDIT e IECPA. Constatou-se que $44 \%$ dos participantes eram consumidores de risco e que $48 \%$ possuíam expectativas positivas altas. Entre elas, facilitação das intersações sociais, diminuição e/ou fuga de emoções negativas, ativação e prazer sexual, efeitos positivos na atividade e humor e na avaliação de si mesmo. Houve correlação entre beber problemático e expectativas positivas. Investigar a relação entre padrão de uso e expectativas sobre os efeitos do álcool favorece o planejamento de intervenções terapêuticas e estratégias preventivas mais precisas que visem a reduzir os riscos do beber problemático entre universitários.
\end{abstract}

Palavras-chave: álcool; expectativas; universitários.

\section{Expectations and Problematic Drinking among College Students}

\begin{abstract}
Problematic drinking is recurrent among college students and is associated with a variety of negative consequences. Therefore, it is important to understand risk factors for this phenomenon. The present study assessed the relationship between expectations over alcohol effects and risk drinking pattern among college students. Participants were 165 college students with average age of 22 years old $(d p=2,5)$ who responded to the AUDIT and IECPA inventories. Results indicated that $44 \%$ of the participants were risk consumers and $48 \%$ had high positive expectations, among them facilitation of social interaction, decrease and/or escape of negative emotions, enhanced sexual pleasure, positive effects on activity, mood and self evaluation. There was a correlation between problematic drinking and positive expectations. To investigate the relationship between pattern of use and expectations over alcohol effects favours planning of therapeutical interventions and more precise preventive strategies aiming at reducing the risks of problematic drinking among college students.
\end{abstract}

Key words: alcohol; expectations; college students.

O período de transição para a universidade tem sido apontado como uma fase de vulnerabilidade aumentada ao uso de álcool e outras drogas. O consumo excessivo de álcool é um padrão recorrente entre universitários (Ham \& Hope, 2003; Park \& Grant, 2005). A elevada incidência do consumo abusivo de álcool entre eles está associada a inúmeras conseqüências negativas tanto para saúde física e mental destes jovens quanto para a sociedade como um todo (Chassin, Pitts \& Prost, 2002; Kerr-Corrêa, Andrade, Bassit \& Boccuto, 1999; O’Malley \& Johnston, 2002; Perkins, 2002; Stempliuk, Barroso, Andrade, Nicastri \& Malbergier, 2005; Windle, 2003; Zeigler \& cols., 2005). Entre os prejuízos relacionados

1 Apoio Financeiro: Conselho Nacional de Desenvolvimento Científico e Tecnológico (CNPq) sob forma de bolsa de Mestrado (primeira autora), bolsa de Iniciação científica (segunda autora) e Produtividade em Pesquisa (terceira autora). Este estudo faz parte da Dissertação de Mestrado em Psicologia (UFRGS) da primeira autora, orientanda da terceira autora.

2 Endereço: Instituto de Psicologia UFRGS, Laboratório de Psicologia Experimental, Neurociências e Comportamento (LPNeC), Rua Ramiro Barcelos 2600, sala 02, Porto Alegre, RS, Brasil 90035-003. E-mail: acpeuker@terra.com.br ao maior consumo de álcool estão morte violenta, exposição a comportamentos de risco (Ex.: dirigir sob efeito do álcool, fazer sexo sem proteção, uso de outras drogas), queda no desempenho acadêmico, prejuízo no desenvolvimento e na estruturação de habilidades cognitivo-comportamentais e emocionais, danos ao patrimônio público e violência (Chassin \& cols., 2002; Ham \& Hope, 2003; O’Malley \& Johnston, 2002; Park \& Grant, 2005; Perkins, 2002; Windle, 2003; Zeigler \& cols., 2005).

Estudos a respeito do consumo de drogas lícitas e ilícitas no Brasil revelam que o consumo de álcool entre jovens alcança prevalências maiores que $60 \%$, podendo alcançar $80 \%$ em alguns estudos. Investigações conduzidas com jovens universitários demonstram índices ainda maiores de consumo de álcool e outras drogas do que aquelas com escolares (Godoi, Muza, Costa \& Gama, 1991; Kerr-Corrêa \& cols., 1999; Sciovoletto, Tsuji \& Abdo, 1999; Tavares, Béria \& Lima, 2001). De acordo com o I Levantamento Domiciliar sobre o Uso de Drogas Psicotrópicas no Brasil (Carlini, Galduróz, Noto \& Nappo, 2002), em relação ao uso na vida de álcool a prevalência é de $48,3 \%$ entre jovens com faixa etária entre 12 e 17 anos, de 107 grandes centros urbanos brasileiros. A análise dos dados desta mesma 
pesquisa, considerando as regiões brasileiras, revela uma maior prevalência de uso na vida de álcool na região Sul $(54,5 \%)$. A cidade de Porto Alegre (RS) é líder no ranking dos usuários regulares de substâncias psicoativas lícitas e ilícitas, com 14,4\% de usuários de álcool (Pechansky, Szobot \& Scivoletto, 2004).

A população universitária apresenta padrões típicos de uso de álcool e fatores de risco, relacionados ao beber problemático, que diferem da população geral (Ex.: normas sociais e comportamentais específicas). Em função disso, é essencial compreender as variáveis que podem estar associadas a este preocupante fenômeno.

Influências sócio-ambientais podem favorecer o consumo excessivo de álcool entre universitários em maior ou menor grau. Por exemplo, uma situação na qual o álcool é amplamente disponível e oferecido ativamente é, obviamente, mais favorecedora do que em um ambiente no qual a oferta não acontece desta forma. A frequiência constante a bares, além de outros fatores, aumenta a probabilidade do uso de drogas, em especial de álcool (Mesquita, Bucaretchi, Castel \& Andrade, 1991). Em geral, nas horas de lazer poucos universitários engajam-se em atividades culturais e/ou esportivas. Em seu tempo livre, geralmente, os estudantes costumam assistir televisão ou sair com amigos. Nestas ocasiões, o comum são idas a bares ou festas onde o uso de álcool é freqüente (Kerr-Corrêa \& cols., 1999). Além disso, universitários expostos a ambientes nos quais o álcool é facilmente obtido e possui baixo custo apresentam maior probabilidade de consumirem álcool excessivamente do que aqueles que não estão expostos a situações desta natureza (Weitzman, Toben, Nelson \& Wechsler, 2003). Bebedores freqüentes (pessoas que bebem no mínimo semanalmente, consomem pelo menos cinco doses de álcool em uma ocasião típica, e ficam bêbadas mensalmente) são mais propensos a beber em contextos nos quais há facilitação social (Ex.: bares, festas) do que bebedores ocasionais (Ham \& Hope, 2003).

O uso de álcool entre universitários também pode ser favorecido de forma indireta. Estudantes influenciamse mutuamente em termos de beber pela modelagem, imitação ou reforçamento do comportamento de beber. A seleção de colegas, a escolha do tipo de substância, o padrão de uso e a forma como o consumo de substâncias de seus pares é percebida parecem interagir neste processo. As normas comportamentais estabelecidas em relação ao beber podem servir para justificar os comportamentos extremados observados entre eles. Sabe-se também que universitários tendem a superestimar tanto a aceitabilidade quanto o comportamento de beber propriamente dito de seus pares. Este viés na percepção de normas de comportamento também pode influenciar os estudantes a engajarem-se em padrões de consumo de álcool de risco. $\mathrm{O}$ indivíduo pode perceber e interpretar o padrão de beber dos outros como um reforçador de seu próprio comportamento e, então, passar a se comportar de acordo com esta percepção (Chassin \& cols., 2002; Dimeff, Baer, Kivlahan \& Marlatt, 2002; Ham \& Hope, 2003; Wechsler, Molnar, Davenport \& Baer, 1999).

A modelagem social parece intensificar-se em um ambiente novo, no qual o indivíduo tem menos experiência e seu repertório comportamental ainda é escasso (Ex.: um calouro em uma festa da faculdade) (Read, Mark \& Capone, 2005; Wood, Read, Palfai \& Stevenson, 2001). Ao ingressar na universidade muitos jovens adultos vivenciam novas experiências como se distanciar da família de origem pela primeira vez, residir com outros estudantes (Ex.: repúblicas), experimentar a ausência da supervisão de adultos (Windle, 2003). Estas experiências novas podem potencializar o uso de álcool e os riscos associados a este consumo. Sendo assim, a entrada na universidade configura-se como um período crítico, de maior vulnerabilidade, para o início e para a manutenção do uso de álcool e outras drogas.

Além de fatores ambientais, expectativas positivas quanto aos efeitos da droga estão associadas ao beber problemático, podendo exercer influências importantes no início e na manutenção do uso desta droga e na emissão de comportamentos relacionados a este consumo (Araujo \& Gomes, 1998; Fromme \& D’Amico, 2000; Goldman, 1999). O desenvolvimento de expectativas de resultado de um comportamento específico resulta da associação aprendida entre estímulos para ação e reforçadores do comportamento. As expectativas (ou cognições antecipatórias) possuem propriedades motivacionais. Desta forma, a apresentação do álcool pode gerar a expectativa de estados afetivos aprendidos associados ao comportamento de beber (Ex.: alegria, prazer) e o desejo de experenciar estas emoções.

As expectativas em relação aos efeitos do álcool são consideradas informações da memória de longo-prazo que derivam de experiências vicárias e diretas que um indivíduo teve com o álcool como conseqüência de suas características biológicas e do ambiente. Expectativas bem definidas a respeito dos efeitos do álcool podem se formar antes mesmo de um indivíduo beber pela primeira vez na vida. Estas expectativas se desenvolvem a partir de modelos familiares e do grupo de iguais, experiências diretas e indiretas com bebidas de álcool e exposição à mídia (Araujo \& Gomes, 1998; Fromme \& D’Amico, 2000; Goldman, 1999).

As expectativas configuram-se como proposições de

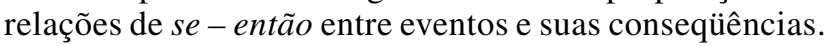
Desta forma, as expectativas de conseqüência podem contribuir para a decisão de beber. Entre as expectativas que podem motivar um indivíduo a beber estão expectativas de maior sociabilidade, aumento e/ou ativação do desejo sexual, redução de tensão, mas também diversas outras. $\mathrm{O}$ conjunto destas diferentes expectativas retrata o comportamento de beber do indivíduo. Por exemplo, expectativas de estados afetivos agradáveis (Ex.: bem-estar, confiança) podem ser confirmadas pela experiência direta com a droga e, então, fortalecer as expectativas previamente existentes. Eventos congruentes com as expectativas são selecionados e registrados. Maior autoconfiança, sociabilidade, desinibição social e atratividade física/sexual estão entre as expectativas correlacionadas ao maior consumo de álcool (Dimeff \& cols., 2002). Bebedores freqüentes mantêm expectativas positivas que geralmente estão incorretas (Wiers, Luitgaarden, Wildenberg \& Smulders, 2005). Há evidência de que bebedores-problema têm mais expec- 
tativas positivas sobre os efeitos imediatos do álcool do que expectativas acerca de suas consequiências negativas a longo-prazo (Ex.: funcionamento social prejudicado) (Lewis \& O'Neill, 2000).

Uma dificuldade comum para avaliar o consumo de álcool de risco entre universitários é a ausência de uma definição operacional padrão do beber problemático. Medidas de quantidade e freqüência do consumo de álcool, de forma isolada, não são suficientes para determinar o status do problema do uso de álcool entre universitários. Por exemplo, alguns bebedores freqüentes podem referir baixos índices de problemas relacionados ao álcool, enquanto alguns bebedores ocasionais ou moderados podem referir altos índices de problemas associados ao beber. Conseqüências negativas relacionadas ao álcool também são um marcador importante para definir o beber problemático entre universitários. Desta forma, medidas de quantidade e freqüência podem servir para avaliar o beber problemático entre universitários, mais acuradamente, quando aliadas à avaliação das conseqüências negativas associadas ao uso de álcool (Ham \& Hope, 2003).

$\mathrm{O}$ risco de desenvolver dependência e de sofrer conseqüências negativas relacionadas ao álcool eleva-se à medida que a freqüência da intoxicação episódica aumenta (Wechsler, Molnar, Davenport \& Baer 1999), especialmente quando há a ocorrência repetida de episódios de intoxicação, denominados binge (Shakeshaft, Bowman \& Sanson-Fisher, 1998; Townshend \& Duka, 2002). Sendo assim, a freqüência de episódios de binge também é um componente importante da definição do beber problemático entre universitários (Ham \& Hope, 2003; Weitzman \& cols., 2003)

Definições sobre o conceito de binge geralmente baseiam-se na quantidade de álcool consumida dentro de um período definido (Ex.: um dia, uma ocasião, número de horas) e na freqüência com que este tipo de consumo acontece (Ex.: semanalmente ou mensalmente). Geralmente, episódios de binge são caracterizados pelo consumo consecutivo de cinco ou mais doses de álcool em uma única ocasião (Ham \& Hope, 2003; Wechsler \& cols., 2002). Entre universitários, observa-se a ampla ocorrência destes episódios, o que torna esta população ainda mais vulnerável aos efeitos deletérios do álcool (Sheffield, Darkes, Del Boca \& Goldman, 2005).

Em função disso, a investigação das expectativas constitui-se como uma ferramenta preventiva útil em função de seu valor preditivo (Pinto Gouveia, Ramalheira, Robalo, Borges \& Rocha-Almeida, 1996). Enquanto representações cognitivas, as expectativas quanto aos efeitos do álcool podem ser modificadas em programas preventivos e paralelamente modificar a frequiência e a quantidade do consumo de álcool. A idéia subjacente é de que bebedores de risco possuem maiores expectativas positivas em relação aos efeitos do álcool, que frequentemente estão incorretas. Assim, a modificação efetiva das expectativas positivas poderia resultar na redução do uso de álcool e nos padrões de consumo de risco (Darkes \& Goldman, 1993; Stacy, Widaman \& Marlatt, 1990; Wiers \& cols., 2005). Além disso, compreender a possível relação entre padrão de consumo e expectativas positivas a respeito dos efeitos do álcool contribui para o planejamento de intervenções terapêuticas e estratégias preventivas mais precisas, visando a reduzir os riscos comportamentais e de saúde associados ao beber problemático entre universitários. Neste contexto, o objetivo deste estudo foi examinar a associação entre expectativas positivas em relação aos efeitos do álcool e o beber problemático em estudantes universitários.

\section{Método}

\section{Participantes}

Estudantes de graduação de diversos cursos da Universidade Federal do Rio Grande do Sul (UFRGS) $(n=165)$ de 22 anos (média, $d p=2,5$ ), destes $51 \%$ eram mulheres $(n=84)$. Os participantes eram provenientes, na maior parte, dos semestres iniciais (entre o $2^{\circ}$ e o $5^{\circ}$ ) dos cursos de Psicologia, Odontologia, Farmácia, Enfermagem e Medicina. O projeto foi aprovado pelo Comitê de Ética em Pesquisa da UFRGS, processo $\mathrm{n}^{\circ} .2005417$.

\section{Instrumentos}

Alcohol Use Disorders Identification Test (AUDIT): este questionário foi elaborado pela Organização Mundial da Saúde (Babor, La Fuente, Saunders \& Grant, 1992). O AUDIT foi traduzido (Figlie, Pillon, Laranjeira \& Dunn, 1997) e validado no Brasil (Méndez, 1999). Este instrumento é composto por 10 questões sobre o uso do álcool que se referem ao último ano, sendo que as três primeiras medem a quantidade e a freqüência do uso regular ou ocasional de álcool, as três questões seguintes investigam sintomas de dependência e as quatro finais referem-se a problemas recentes na vida relacionados ao consumo (Bergman \& Källmén, 2002). O escore final pode variar de zero a 40 pontos. Este é um método que identifica pessoas com consumo de risco, uso nocivo e dependência do álcool (Henrique, Micheli, Lacerda, Lacerda \& Formigoni, 2004; Mendoza-Sassi \& Béria, 2003). Neste estudo, o padrão de risco de uso de álcool, ou beber problemático, foi definido pelo escore $\geq 8$ no AUDIT, conforme estudos prévios conduzidos no Brasil (Henrique \& cols., 2004; Mendoza-Sassi \& Béria, 2003). $\mathrm{O}$ padrão binge, também considerado um padrão de consumo de risco, foi caracterizado neste estudo pela resposta à terceira questão do AUDIT (Shakeshaft \& cols., 1998). $\mathrm{O}$ item três do AUDIT corresponde à pergunta: "Qual a frequiência que você consome seis ou mais doses de bebida alcoólica em uma ocasião?". As respostas possíveis são "nunca", "menos que mensalmente", "mensalmente", "semanalmente" ou "diariamente ou quase diariamente". Os sujeitos que selecionaram qualquer resposta diferente de "nunca" foram caracterizados como bebedores com padrão binge (Shakeshaft \& cols., 1998).

Inventário de Expectativas e Crenças Pessoais acerca do Álcool (IECPA): O inventário desenvolvido por Pinto Gouveia e colaboradores teve sua versão brasileira adaptada por Blanca Guevara Werlang e Margareth da Silva Oliveira (Pinto Gouveia \& cols., 1996). Trata-se de uma medida escalar, de tipo Likert, com 61 itens. Cada item 
consiste numa afirmação sobre expectativas e crenças a respeito dos efeitos do álcool distribuídas em cinco fatores. Os cinco fatores que integram o IECPA incluem efeitos globais positivos ("beber me deixa mais corajoso") e facilitação das interações sociais ("o álcool me torna mais alegre e simpático"), diminuição e/ou fuga de emoções negativas ("o álcool me faz esquecer os problemas da vida"), ativação e prazer sexual ("tenho mais prazer sexual após ter bebido"), efeitos positivos na atividade e humor ("quando bebo aprecio melhor as coisas boas da vida") e na avaliação de si mesmo ("quando bebo fico menos nervoso"). Para cada item são apresentadas cinco alternativas de resposta: Não concordo, Concordo pouco, Concordo moderadamente, Concordo muito e Concordo muitíssimo, que recebem escores de 1 a 5 , respectivamente. O escore final varia de zero a 305 pontos. A pressuposição subjacente é a de que maiores escores no IECPA caracterizam participantes com expectativas positivas mais altas e, portanto, maior vulnerabilidade ao alcoolismo. Se o indivíduo é da população geral, o ponto de corte é de 121,82 . Ou seja, aqueles que apresentarem escore total de 122 ou mais têm probabilidade de serem ou virem a se tornar dependentes de álcool (Pinto Gouveia \& cols., 1996).

\section{Procedimentos}

Os participantes foram abordados diretamente no Campus Saúde da UFRGS nos intervalos ou durante as aulas. Todos os indivíduos que consentiram em participar da pesquisa assinaram o Termo de Consentimento Livre e Esclarecido. Os questionários AUDIT e IECPA foram administrados em sessões individuais. Os instrumentos de coleta foram distribuídos em envelopes codificados, a fim de garantir o sigilo e a confidencialidade dos dados.

\section{Análise dos dados}

Primeiramente, os dados foram submetidos aos procedimentos de estatística descritiva para avaliar as variáveis estudadas em termos de distribuição de freqüências, escores, médias e desvio padrão. Para a análise subsequiente dos dados, foi utilizada estatística inferencial, incluindo teste de correlação linear e o teste $T$ de Student. O pacote estatístico utilizado foi o SPSS (Statistical Package for the Social Sciences).

\section{Resultados}

No AUDIT, 44,2\% dos participantes foram caracterizados como consumidores de risco (escore maior ou igual a 8), dentre os quais $35,7 \%$ das mulheres e $53,1 \%$ dos homens. Se o ponto de corte para consumo de risco em mulheres fosse modificado para escore $\geq 6$ o número de bebedores problemáticos aumentaria para $54 \%$ (55\% das mulheres versus $53 \%$ dos homens). A média dos escores do total de participantes no AUDIT foi 7,21 $(d p=4,7)$. Através do teste $T$ de Student para amostras independentes não foram constatadas diferenças entre os sexos no AUDIT ( $t=-1,25 ; g l=163 ; p=0,56$ ).

A Tabela 1 apresenta a distribuição das respostas no AUDIT. Em relação à frequiência do consumo de álcool,
$45,5 \%$ dos universitários referiram beber de duas a quatro vezes por mês, $17 \%$ deles bebiam de duas a três vezes por semana e $6 \%$ deles consumiam álcool quase todos os dias. No que concerne à quantidade do consumo de álcool em um dia típico, 29,7\% reportaram beber de três a quatro doses, $23,6 \%$ deles consumiam de cinco a seis doses, $10,3 \%$ de sete a nove doses e $4,8 \%$ referiram beber 10 ou mais doses em uma ocasião típica de consumo de álcool.

Do total de participantes, $67,8 \%$ revelaram ter consumido seis ou mais doses de álcool em uma única ocasião (binge). Considerando a freqüência dos episódios de binge (questão 3 do AUDIT), 20\% dos universitários afirmaram que estes episódios aconteciam mensalmente e $13,3 \%$ dos participantes experenciaram episódios de binge semanalmente.

Considerando as três questões do AUDIT que investigam sintomas de dependência (números 4, 5 e 6), observa-se que

Tabela 1. Distribuição das respostas AUDIT entre universitários $(n=165)$.

\begin{tabular}{|c|c|}
\hline Item & $\%$ \\
\hline \multicolumn{2}{|l|}{1 - Drinks contendo álcool } \\
\hline Nunca & 09,7 \\
\hline Mensalmente ou menos & 27,3 \\
\hline 2 a $4 x$ por mês & 45,5 \\
\hline 2 a $3 x$ por semana & 17,0 \\
\hline 4 ou mais vezes por semana & 06,0 \\
\hline \multicolumn{2}{|c|}{2 - Número de Drinks num dia típico } \\
\hline zero & 10,3 \\
\hline $1-2$ & 21,2 \\
\hline $3-4$ & 29,7 \\
\hline $5-6$ & 23,6 \\
\hline $7-9$ & 10,3 \\
\hline 10 ou mais & 04,8 \\
\hline \multicolumn{2}{|l|}{3 - Freqüência de 6 ou mais Drinks } \\
\hline Nunca & 32,1 \\
\hline Menos que mensalmente & 34,5 \\
\hline Mensalmente & 20,0 \\
\hline Semanalmente & 13,3 \\
\hline Diariamente o quase diariamente & 0 \\
\hline \multicolumn{2}{|c|}{4 - Perda do controle para parar de beber } \\
\hline Nunca & 78,2 \\
\hline Menos que mensalmente & 17,5 \\
\hline Mensalmente & 04,8 \\
\hline Semanalmente & 02,4 \\
\hline Diariamente o quase diariamente & 0 \\
\hline \multicolumn{2}{|c|}{5 - Falha em fazer o que era esperado devido ao beber } \\
\hline Nunca & 74,5 \\
\hline Menos que mensalmente & 22,4 \\
\hline Mensalmente & 03,0 \\
\hline Semanalmente & 0 \\
\hline Diariamente o quase diariamente & 0 \\
\hline
\end{tabular}




\begin{tabular}{lc}
\hline 6 - Necessidade de uma primeira dose pela manhã & \\
Nunca & 98,2 \\
Menos que mensalmente & 01,8 \\
Mensalmente & 0 \\
Semanalmente & 0 \\
Diariamente o quase diariamente & 0 \\
\hline 7 - Remorso ou culpa depois de beber & \\
Nunca & 64,2 \\
Menos que mensalmente & 29,7 \\
Mensalmente & 04,8 \\
Semanalmente & 01,2 \\
Diariamente o quase diariamente & \\
\hline 8 - Frequiência de apagamentos & \\
Nunca & 63,0 \\
Menos que mensalmente & 29,1 \\
Mensalmente & 06,1 \\
Semanalmente & 01,8 \\
Diariamente o quase diariamente & 0 \\
\hline 9. Criticas pelos resultados de suas bebedeiras & \\
Nunca & \\
Menos que mensalmente & 57,6 \\
Mensalmente & 36,4 \\
Semanalmente & 03,6 \\
Diariamente o quase diariamente & 02,4 \\
\hline 10 - Conselhos de terceiros para parar de beber & 0 \\
Nunca & \\
Menos que mensalmente & 01,8 \\
Mensalmente & 0 \\
Semanalmente & \\
Diariamente o quase diariamente & \\
\hline
\end{tabular}

21,7\% dos universitários revelaram já ter tido a percepção de não conseguir parar de beber uma vez que tinham começado. Destes, 4,8\% tiveram esta percepção mensalmente e 2,4\% semanalmente. Verificou-se ainda que $25,4 \%$ dos universitários já haviam deixado de fazer o que era esperado devido ao consumo de álcool. Além disso, 1,8\% revelaram ter precisado de uma primeira dose pela manhã para sentirem-se melhor depois de uma bebedeira.

Em relação às quatro questões finais do AUDIT que dizem respeito a problemas recentes na vida relacionados ao consumo (números 7, 8, 9 e 10), constatou-se que 35,7\% dos universitários já haviam sentido culpa ou remorso depois de beber, sendo que $6 \%$ arrependeram-se mensal ou semanalmente de ter bebido. Apagamentos (blackouts) ocorreram em $37 \%$, sendo que $6,1 \%$ tiveram blackouts mensalmente e $1,8 \%$, semanalmente. Foi constatado que $42,4 \%$ dos universitários foram criticados pelo resultado de suas bebedeiras. Quanto à frequiência destas críticas, para 36,4\% dos universitários elas aconteceram menos que mensalmente, para 3,6\% mensalmente e $2,4 \%$ receberam críticas semanalmente. Por fim, nas respostas à questão 10 do AUDIT, 13,3\% dos participantes referiram ter sido aconselhados a parar de beber.

No IECPA, observou-se que 47,9\% do total de participantes possuíam altas expectativas positivas em relação aos efeitos do álcool, isto é, apresentaram escore maior ou igual a 122. O escore médio do total de participantes no IECPA foi de $124,73(\underline{d p}=47,2)$. Em relação ao sexo, $44 \%$ das mulheres $(n=37)$ e $51,9 \%$ dos homens $(n=42)$ apresentaram altas expectativas positivas em relação aos efeitos do álcool (escore $\geq 122$ ). Considerando o teste $T$ de Student para amostras independentes, não foram observadas diferenças entre os sexos em relação ao IECPA $(t=-0,76 ; g l=163 ; p=0,86)$. Além disso, no que concerne aos cinco fatores que compõem o IECPA (ver Instrumentos) também não foram constatadas diferenças estatísticas entre os sexos. Por fim, observou-se, através do coeficiente de correlação de Pearson, uma associação positiva entre os escores do IECPA e os do AUDIT $(r=0,6 ; p<0,001)$.

\section{Discussão}

Constatou-se que $44,2 \%$ dos participantes poderiam ser caracterizados como bebedores-problema (escore $\geq 8$ ), sendo $35,7 \%$ das mulheres e $53,1 \%$ dos homens. Se o ponto de corte para consumo de risco em mulheres fosse modificado para escore $\geq 6$, como sugerido na literatura em função das diferenças biológicas entre os sexos (Bergman \& Källmén, 2002), a porcentagem de bebedores-problema aumentaria para $54 \%$.

O percentual de bebedores-problema medido pelo AUDIT foi superior àqueles encontrados em estudos prévios conduzidos no Brasil. Em uma investigação de base populacional realizada em Rio Grande (RS), foi observada uma prevalência de 7,9\% de bebedores-problema (escore $\geq 8$ ), com 14,5\% de homens e 2,4\% de mulheres (Mendoza-Sassi \& Béria, 2003). Uma pesquisa realizada por Figlie, Pillon, Dunn e Laranjeira (2000) em um hospital geral, com 275 pacientes de ambos os sexos, utilizando o mesmo ponto de corte, revelou que 34 eram bebedores-problema, sendo $30(22 \%)$ homens e quatro (3\%) mulheres. Sendo assim, o beber problemático entre os participantes do presente estudo é duas ou três vezes mais freqüente entre os homens e mais de 10 vezes mais freqüente entre as mulheres do que o esperado na população geral. Além disso, não foi encontrada diferença entre os sexos no que concerne ao beber problemático entre universitários. Este fato pode refletir o aumento no consumo de bebidas alcoólicas entre as mulheres. Em outros países, tem sido reportado um aumento na incidência do uso de álcool entre as mulheres, principalmente entre as jovens (Hartley, Elsabagh \& File, 2004; Wechsler \& cols., 2002; Young, Morales, McCabe, Boyd \& Darcy, 2005).

É importante salientar que, embora a versão do AUDIT validada no Brasil tenha uma boa sensibilidade $(91,8 \%)$, a especificidade $(62,3 \%)$ do teste é baixa (Méndez, 1999). Portanto, é possível que uma parcela da amostra caracterizada como bebedores-problema representem casos falsos positivos. Entretanto, os resultados obtidos com esta mesma escala na população geral estariam igualmente superestimados. Apesar disso, os resultados obtidos revelam a ampla 
ocorrência do padrão de risco de uso de álcool e de altas expectativas em relação aos seus efeitos entre os universitários. Estes comportamentos podem se perpetuar na vida adulta e serem indicativos da necessidade de desenvolver estratégias preventivas específicas a esta população.

Quanto ao consumo de álcool, 38,7\% deles referiram consumir pelo menos cinco doses em uma ocasião típica de consumo de álcool. Este percentual revela que quase $40 \%$ dos participantes apresentam o padrão binge de uso de álcool, caracterizado pelo consumo consecutivo de cinco ou mais doses de álcool em uma única ocasião. Isto se confirma considerando as respostas à questão três do AUDIT, pois aproximadamente $70 \%$ dos participantes afirmaram beber seis ou mais drinks por ocasião. Além disso, constatou-se que os episódios de binge aconteciam regularmente, para $33,3 \%$ dos universitários.

$\mathrm{O}$ padrão binge enquadra-se dentro do beber problemático. A ocorrência repetida de episódios de binge está vinculada à maior freqüência de comportamentos de risco (Ex.: atividade sexual não planejada e sem proteção, dirigir embriagado, uso de tabaco, tentativas de suicídio) e de consequiências negativas relacionadas ao álcool (dificuldades acadêmicas, problemas com a polícia, gravidez não desejada, doenças sexualmente transmissíveis) (Park \& Grant, 2005; Sheffield \& cols., 2005; Wechsler \& cols., 2002; Windle, 2003). Um estudo norte-americano constatou que, entre universitários que relataram ter dirigido depois de beber, $41 \%$ foram classificados como bebedores com episódios de binge freqüentes (Wechsler \& cols., 1999). Além disso, períodos de abstinência seguidos por episódios de consumo exacerbado de álcool podem aumentar o risco de desenvolver dependência desta substância e prejuízos neuropsicológicos (Townshend \& Duka, 2002). Alguns autores sugerem que o padrão binge, mais do que a ingestão de álcool por si, é responsável por prejuízos no desempenho de tarefas cognitivas associadas ao funcionamento do lobo frontal, como as funções executivas (Hartley \& cols., 2004; Weissenborn \& Duka, 2003).

Episódios de binge também podem causar apagamento ou perda da memória para eventos que ocorreram durante a ocasião de intoxicação alcoólica (blackouts). Em relação à ocorrência de apagamentos, os universitários com padrão binge de uso de álcool apresentaram uma freqüência de apagamentos cinco vezes maior $(50 \%)$ do que aqueles que não apresentavam este padrão $(9,4 \%)$. O número de bebedores com padrão binge pode ter sido subestimado porque a questão três do AUDIT estabelece uma quantidade determinada de doses por ocasião, não levando em conta as diferenças de homens e mulheres quanto à distribuição, metabolização e efeitos do álcool entre os sexos (Ham \& Hope, 2003; Oei \& Morawska, 2004; Shakeshaft \& cols., 1998).

Considerando as questões do AUDIT que investigam sintomas de dependência (números 4, 5 e 6), alguns aspectos merecem destaque. Observou-se que $21,7 \%$ dos universitários experimentaram a sensação de perda do controle para parar de beber. Deste total, 7,2\% não conseguiam, de forma regular, parar de beber uma vez que tinham começado. $\mathrm{Na}$ população geral, a sensação de perda do controle não chega a $6 \%$ das pessoas e apenas $2,4 \%$ têm esta sensação regularmente. Sendo assim, os índices na população investigada são três vezes maiores do que aqueles observados na população geral. Verificou-se ainda que 25,4\% dos universitários deixaram de fazer o que era esperado devido ao consumo de álcool. Na população geral, este percentual é quase oito vezes menor (Mendoza-Sassi \& Béria, 2003).

Em relação às quatro questões finais do AUDIT que dizem respeito a problemas recentes na vida relacionados ao consumo (números 7, 8, 9 e 10) novamente evidencia-se a ocorrência do beber problemático na população investigada. Um montante de $35,7 \%$ dos universitários já havia sentido culpa ou remorso depois de beber. Na população geral, o número de pessoas que sente remorso ou culpa depois de beber é quase cinco vezes menor do que o encontrado entre os universitários pesquisados (Mendoza-Sassi \& Béria, 2003).

Quanto à ocorrência de apagamentos, um total de $37 \%$ dos universitários relatou ter tido algum episódio de apagamento (blackout) por causa do uso de álcool. Na população geral, a freqüência de apagamentos é de 7,7\%, ou seja, quase cinco vezes menor do que entre os participantes do estudo. Para $7,9 \%$ deles estes episódios de perda de memória se davam regularmente, o dobro do percentual encontrado na população geral (Mendoza-Sassi \& Béria, 2003). A ocorrência de apagamentos está baseada na quantidade de álcool consumida em uma ocasião. Em função disso, bebedores com padrão binge de uso de álcool, incluindo estudantes universitários, tendem a apresentar maior frequiência de apagamentos (Zeigler \& cols., 2005).

No IECPA, observou-se que, quase a metade $(47,9 \%)$ do total de participantes, apresentava altas expectativas positivas em relação aos efeitos do álcool. Isto é, considerando este padrão alto de expectativas que eles mantêm, aproximadamente $50 \%$ dos participantes têm probabilidade de serem ou virem a ser dependentes do álcool. Quanto ao sexo, tanto as mulheres $(44 \%)$ quanto os homens $(51,9 \%)$ apresentaram altas expectativas positivas em relação aos efeitos do álcool. Entre as quais: facilitação das interações sociais, diminuição e/ou fuga de emoções negativas, ativação e prazer sexual, efeitos positivos na atividade e humor e na avaliação de si mesmo. De acordo com isso, em uma investigação com 367 universitários norte-americanos, de ambos os sexos, observou-se uma alta correlação entre consumo freqüente e a crença de que o álcool facilitaria o desempenho de atividades sexuais (Larimer, 1997). Em outro estudo, constatou-se que estudantes com maior expectativa de que o consumo de álcool poderia aumentar a confiança em situações sociais ou aliviar a tensão estavam mais propensos a reportarem problemas sócio-emocionais mais graves, como depressão, ansiedade, dificuldades de relacionamento familiar e/ou com os outros e sentimentos negativos sobre si mesmos. Além disso, estes estudantes também tinham maior probabilidade de relatar efeitos agudos do uso de álcool (náusea, vômito), gastar mais dinheiro com álcool ou outras drogas, dirigir sob influência de drogas e apresentar problemas com a lei (O'Hare \& Sherrer, 1997). Em concordância com estes resultados, foi demonstrado que calouros universitários com altos níveis de expectativas de aumento da sociabilidade tinham mais chance de experenciar consequiências negativas decorrentes do beber problemático, tais como: apagamentos, ressaca e envolvimento em brigas (Turrisi, Wiersma, \& Hughes, 2000). Sendo assim, expectativas positivas e distorcidas ou falsas 
crenças quanto aos efeitos do álcool podem estar associadas tanto ao maior consumo alcoólico como também à ocorrência de consequiências negativas.

Apesar destes resultados não poderem ser extrapolados para populações clínicas ou participantes com menor nível educacional, eles apresentam uma importante contribuição para o entendimento do beber problemático em universitários no contexto cultural brasileiro. Por se tratar de um estudo transversal não foram estabelecidas relações causais, mas apenas associações descritivas, cujos vetores de causalidade podem estar em pontos desconhecidos pelos pesquisadores. Fatores individuais, de personalidade, familiares, genéticos, entre outros podem ser relevantes para a compreensão do beber entre universitários. Portanto, sugere-se que investigações futuras utilizem delineamentos longitudinais que permitam avaliar o fenômeno do beber problemático entre universitários em mais de um momento (Ex.: antes da entrada e após o ingresso na universidade), além da inclusão de outras variáveis pertinentes.

A correlação entre expectativas positivas e o beber problemático sugere que programas preventivos para reduzir os riscos do beber problemático entre universitários devem alcançar pelo menos dois aspectos. $\mathrm{O}$ aspecto ambiental, objetivando limitar a propaganda, o acesso e a disponibilidade do álcool, aumentar seu custo e promover atividades alternativas não relacionadas ao uso de álcool (Kerr-Corrêa \& cols., 1999; Zeigler \& cols., 2005); e o aspecto cognitivo, objetivando identificar e alterar cognições disfuncionais. Programas baseados no desenvolvimento e treinamento de habilidades cognitivo-comportamentais e estratégias motivacionais para bebedores-problema têm produzido resultados positivos - em especial, redução de conseqüências negativas relacionadas ao álcool e modificação do beber problemático (Borsari \& Carey, 2000; Dimeff \& cols., 2002).

A ingestão persistente de álcool é particularmente prejudicial no período da adolescência e idade adulta jovem. Entre as tarefas desenvolvimentais destes períodos está a contínua integração de habilidades cognitivas assim como a formação das habilidades necessárias para o funcionamento bem sucedido do papel adulto. Neste sentido, os resultados obtidos refletem a necessidade de desenvolver estratégias preventivas específicas a esta população (Ex. disponibilizar informação científica, detectar precocemente o uso de drogas). Muitos jovens ingressam na universidade em idade e circunstâncias propícias à aquisição de novas competências. Desta forma, o ambiente acadêmico torna-se um espaço adequado para o desenvolvimento de programas preventivos, sendo recomendável a implantação de políticas nessa direção.

\section{Referências}

Araujo, L. B. \& Gomes, W. B. (1998). Adolescência e as expectativas em relação aos efeitos do álcool. Psicologia: Reflexão e Crítica, 11(1), 05-33.

Babor, T. F., La Fuente, J. R., Saunders, J. \& Grant, M. (1992). AUDIT: The alcohol use disorders identificacion test: guidelines for use in primary health care. WHO (World Health Organization)/PSA, 4, 1-29.
Bergman, H. \& Källmén, H. (2002). Alcohol use among Swedes and psychometric evaluation of the alcohol use disorders identification test. Alcohol \& Alcoholism, 37, 245-251.

Borsari, B. \& Carey, K. B. (2000). Effects of a brief motivacional intervention with college student drinkers. Journal of Consulting and Clinical Psychology, 68, 728-733.

Carlini, E. A., Galduróz, J. C. F., Noto, A. R. \& Nappo, S. A. (2002). I levantamento domiciliar sobre o uso de drogas psicotrópicas no Brasil: estudo envolvendo as 107 maiores cidades do país 2001. São Paulo: Centro Brasileiro de Informações sobre Drogas Psicotrópicas, Universidade Federal de São Paulo.

Chassin, L., Pitts, S. C. \& Prost, J. (2002). Binge drinking trajectories from adolescent to emerging adulthood in a high-risk sample: Predictors a substance abuse outcomes. Journal of Consulting and Clinical Psychology, 70, 67-78.

Darkes, J. \& Goldman, M. S. (1993). Expectancy challenge and drinking reduction: Experimental evidence for a mediational process. Journal Consulting of Clinical Psychology, 52, 639650.

Dimeff, L. A., Baer, J. S., Kivlahan \& Marlatt, G. A. (2002). Alcoolismo entre estudantes universitários: Uma abordagem de redução de danos. São Paulo: UNESP.

Figlie, N. B., Pillon, S. C., Laranjeira, R. \& Dunn, J. (1997). AUDIT identifica a necessidade de interconsulta específica para dependentes de álcool no Hospital Geral? Jornal Brasileiro de Psiquiatria, 46, 589-593.

Figlie, N.B., Pillon, S. C., Dunn, J. \& Laranjeira, R. (2000). The frequency of smoking and problem drinking among general hospital inpatients in Brazil- using the AUDIT and Fagerström questionnaires. Revista Paulista de Medicina, 5, 139-143.

Fromme, K. \& D'Amico, E. J. (2000). Measuring adolescent alcohol outcome expectancies. Psychology of Addictive Behaviors, 14, 206-212.

Godoi, A. M., Muza, G. M., Costa, M. P. \& Gama, M. T (1991). Consumo de substâncias psicoativas entre estudantes da rede privada. Revista de Saúde Pública, 25, 150-156.

Goldman, M. S. (1999). Risk for substance abuse: Memory as a common etiological pathway. Psychological Science, 10, 196-19.

Ham, L. S. \& Hope, D. A. (2003). College students and problematic drinking: A review of the literature. Clinical Psychology Review, 23, 719-759.

Hartley, D. E., Elsabagh, S. \& File, S. E. (2004). Binge drinking and sex: Effects on mood and cognitive function in healthy young volunteers. Pharmacology, Biochemistry and Behavior, 78, 611-619.

Henrique, I. F. S., De Micheli, D., Lacerda, R. B.; Lacerda, L. A. \& Formigoni, M. L. O. S. (2004). Validation of the Brazilian version of Alcohol, Smoking and Substance Involvement Screening Test (ASSIST). Revista da Associação Médica Brasileira, 50, 199-206.

Kerr-Corrêa, F., Andrade, A. G., Bassit, A. Z. \& Boccuto, N. M. V. F. (1999). Uso de álcool e drogas por estudantes de medicina da Unesp. Revista Brasileira de Psiquiatria, 21, 95-100.

Larimer, M. E. (1997). College drinking and the Greek system: examining the role of perceived norms for high-risk behavior. Journal of College Student Development, 38, 587-598.

Lewis, B. A. \& O'Neill, H. K. (2000). Alcohol expectancies and social deficits relating to problem drinking among college students. Addictive Behaviors, 25, 295-299. 
Méndez, E.B. (1999). Uma versão brasileira do AUDIT- Alcohol Use Disorders Identification Test. Dissertação de Mestrado, Universidade Federal de Pelotas, Pelotas.

Mendoza-Sassi, R. A. \& Béria, J. U. (2003). Prevalence of alcohol use disorders and associated factores: a population-based study using AUDIT in southern Brazil. Addiction, 98, 799-804.

Mesquita, A. M. C., Bucaretchi, H. A., Castel, S. \& Andrade, A. G. (1991). Estudantes da Faculdade de Medicina de São Paulo: uso de substâncias psicoativas em 1991. Revista Brasileira de Psiquiatria, 17, 47-54.

Oei, T. P. S. \& Morawska, A. (2004). A cognitive model of binge drinking: The influence of alcohol expectancies and drinking refusal self-efficacy. Addictive Behaviors, 29, 159-179.

O'Hare, T. \& Sherrer, M. (1997). Drinking problems, alcohol expectancies, and drinking contexts in college first offenders. Journal of Alcohol and Drug Education, 43, 31-45.

O'Malley, P. M. \& Johnston, L. D. (2002). Epidemiology of alcohol and other drug use among American college students. Journal of Studies on Alcohol, Suppl., 2002 Mar, 14, 23-39. Review.

Park, C.L. \& Grant, C. (2005). Determinants of positive e negative consequences of alcohol consumption in college students: alcohol use, gender e psychological characteristics. Addictive Behaviors, 30, 755-765.

Pechansky, F., Szobot, C. M. \& Scivoletto, S. (2004). Uso de álcool entre adolescentes: Conceitos, características epidemiológicas e fatores etiopatogêncios. Revista Brasileira de Psiquiatria, 26, 14-17.

Perkins, H. W. (2002). Surveying the damage: a review of research on consequences of alcohol misuse in college populations. Journal of Studies on Alcohol, Suppl., 2002 Mar, 14, 91-100.

Pinto Gouveia, J., Ramalheira, C., Robalo, M. T., Borges, J. C. \& Rocha-Almeida, J. (1996). Inventário de expectativas e crenças pessoais acerca do álcool (IECPA) (Versão brasileira). São Paulo: Casa do Psicólogo.

Read, J. P., Mark, D. W. \& Capone, C. (2005). A prospective investigation of relations between social influences and alcohol involvement during the transition into college. Journal of Studies on Alcohol, 66, 23-34.

Sciovoletto, S., Tsuji, R. \& Abdo, C. H. (1999). Relação entre o consumo de drogas e comportamento sexual de estudantes de $2^{\circ}$. Grau de São Paulo. Revista Brasileira de Psiquiatria, 21, 28-32.

Shakeshaft, A. P., Bowman, J. A. \& Sanson-Fisher, R. W. (1998). Comparison of three methods to assess binge consumption: One-week retrospective drinking diary, AUDIT, and quantity/ frequency. Substance Abuse, 19, 191-203.

Sheffield, F. D., Darkes, J., Del Boca, F. K. \& Goldman, M. S. (2005). Binge drinking and alcohol-related problems among community college students: implications for prevention policy. Journal of American College Health, 54, 137-141.

Stacy, A. W., Widaman, K. F. \& Marllat, G. A. (1990). Expectancy models of alcohol use. Journal of Personality and Social Psychology, 58, 918-928.
Stempliuk, V. A, Barroso, L. P., Andrade, A. G., Nicastri, S. \& Malbergier, A (2005). Comparative study of drug use among undergraduate at the university of São Paulo - São Paulo campus in 1996 and 2001. Revista Brasileira de Psiquiatria, 27, 185-193.

Tavares, B. F., Béria, J. U. \& Lima, M. S. (2001). Prevalência do uso de drogas e desempenho escolar entre adolescentes. Revista de Saúde Pública, 35, 150-158.

Townshend, J. M. \& Duka, T. (2002). Patterns of alcohol drinking in a population of young social drinkers: a comparison of questionnaire and diary measures. Alcohol \& Alcoholism, 37, 187-192.

Turrisi, R., Wiersma, K. A. \& Hughes, K. K. (2000). Binge-drinkingrelated consequences in college students: role of drinking beliefs and mother-teen communications. Psychology of Addictive Behaviors, 14, 342-355.

Wechsler, H., Lee, J. E., Meichun, K., Seibring, M., Toben, N. \& Lee, H. (2002). Trends in college binge drinking during a period of increased prevention efforts. Findings from 4 Harvard school of public health college alcohol study surveys: 1993-2001. Journal of American College Health, 50, 203-217.

Wechsler, H., Molnar, B. E., Davenport, A. E. \& Baer, J. (1999). College alcohol use: a full or empty glass? Journal of American College Health, 47, 247-252.

Weissenborn, R. \& Duka, T. (2003) Acute alcohol effects on cognitive function in social drinkers: their relationship to drinking habits. Psychopharmacology, 165, 306-312.

Weitzman, E. R., Toben, F. N. \& Wechsler, H. (2003). Taking up binge drinking in college: the influences of person, social group, and environment. Journal of Adolescent Health, 32, 26-35.

Wiers, R. W., Luitgaarden, J. V., Wildenberg, E. V. \& Smulders, F. T. (2005). Challenging implicit and explicit alcohol-related cognitions in young heavy social drinkers. Addiction, 100, 806-819.

Windle, M. (2003). Alcohol use among adolescents and young adults. Alcohol Research \& Health, 27, 79-85.

Wood, M. D., Read, J. P., Palfai, T. P. \& Stevenson, J. F. (2001). Social influence processes and college student drinking: The mediational role of outcome expectancies. Journal of Studies on Alcohol, 62, 32-43.

Young, A. M., Morales, M., McCabe, S. E., Boyd, C. J. \& Darcy, H. (2005). Drinking like a guy: frequent binge drinking among undergraduate women. Substance Use and Misuse, 40, 241267.

Zeigler, D. W., Wang, C. C., Yoast, R. A., Dickinson, B. D., McCafrree, M. A., Robinowitz, C. B. \& Sterling, M. L. (2005). The neurocogntive effects of alcohol on adolescents and college students. Preventive Medicine, 40, 23-32.s

Recebido em 14.02.2006

Primeira decisão editorial em 03.04.2006

Versão final em 10.05.2006

Aceito em 05.06.2006 